Purdue University

Purdue e-Pubs

CTRC Research Publications

Cooling Technologies Research Center

2013

\title{
Evaporative Heat Transfer from an Electrowetted Liquid Ribbon on a Heated Substrate
}

Chris P. Migliaccio

Purdue University

S V. Garimella

Purdue University, sureshg@purdue.edu

Follow this and additional works at: http://docs.lib.purdue.edu/coolingpubs

Migliaccio, Chris P. and Garimella, S V., "Evaporative Heat Transfer from an Electrowetted Liquid Ribbon on a Heated Substrate" (2013). CTRC Research Publications. Paper 181.

http://dx.doi.org/http://dx.doi.org/10.1016/j.ijheatmasstransfer.2012.10.009

This document has been made available through Purdue e-Pubs, a service of the Purdue University Libraries. Please contact epubs@purdue.edu for additional information. 


\title{
Evaporative Heat Transfer from an Electrowetted Liquid Ribbon on a Heated Substrate
}

\author{
Christopher P. Migliaccio and Suresh V. Garimella ${ }^{\text {If }}$ \\ Cooling Technologies Research Center, an NSF IUCRC \\ School of Mechanical Engineering and Birck Nanotechnology Center \\ Purdue University \\ West Lafayette, IN 47907-2088 USA
}

\begin{abstract}
Evaporation of narrow water ribbons (of 5 and $7 \mu \mathrm{L}$ volume) formed on a heated surface is investigated. Chemical and structural patterning of a silicon substrate is employed to fabricate a hydrophilic stripe that bisects hydrophobic pillar arrays of varied geometric roughness. Electrical heating of a $100 \mathrm{~nm}$ titanium layer on the back side of the device provides a constant heat flux. In the absence of electrical actuation, water introduced onto the substrate takes a bulging ribbon shape that is constrained to the immediate vicinity of the hydrophilic stripe. Electrowetting of the water ribbon spreads it into the hydrophobic pillar arrays on either side, leading to significant increases in maximum wetted width (up to 200\%) and wettability (up to $80 \%$ reduction in contact angle). Infrared thermography is employed to characterize the cooling effect due to the spreading of the ribbon, while a goniometer monitors the ribbon shape. The heat transfer in each case is estimated through an energy balance analysis, and the results are compared with other electrowetting-based cooling techniques.
\end{abstract}

Keywords: Liquid ribbon, droplet, electrowetting, evaporation, electronics cooling

\footnotetext{
${ }^{\mathbb{T}}$ Author to whom correspondence should be addressed: 765-494-5621, sureshg@purdue.edu
} 


\section{Nomenclature}

\begin{tabular}{|c|c|c|c|}
\hline$\vec{A}$ & control volume face area vector & Greek & \\
\hline$A$ & area $\left(\mathrm{mm}^{2}\right)$ & $\alpha$ & central angle (rad) \\
\hline$a$ & pillar width $(\mu \mathrm{m})$ & $\beta$ & constant used in Eq. 15 (W) \\
\hline$b$ & pillar pitch $(\mu \mathrm{m})$ & $\delta$ & device thickness $(\mu \mathrm{m})$ \\
\hline$C$ & chord length (mm) & $\varepsilon$ & emissivity \\
\hline$c_{p}$ & specific heat $(\mathrm{J} / \mathrm{kg} \mathrm{K})$ & $\theta$ & apparent contact angle $\left({ }^{\circ}\right)$ \\
\hline$E$ & energy $(\mathrm{J})$ & $\rho$ & density $\left(\mathrm{kg} / \mathrm{m}^{3}\right)$ \\
\hline$H$ & segment height (mm) & $\sigma$ & $\begin{array}{l}\text { Boltzmann constant }\left(5.67 \times 10^{-8}\right. \\
\left.\mathrm{W} / \mathrm{m}^{2} \mathrm{~K}^{4}\right)\end{array}$ \\
\hline$h$ & convection coefficient $\left(\mathrm{W} / \mathrm{m}^{2} \mathrm{~K}\right)$ & $\phi$ & $\begin{array}{l}\text { ratio of pillar tip surface area to } \\
\text { footprint area }\end{array}$ \\
\hline$h_{f g}$ & latent heat $(\mathrm{J} / \mathrm{kg})$ & & \\
\hline$l$ & length (mm) & Subscri & \\
\hline$P$ & perimeter $(\mathrm{mm})$ & avg & average \\
\hline$q$ & heat $(\mathrm{W})$ & bot & bottom surface of device \\
\hline$q^{\prime \prime}$ & heat flux $\left(\mathrm{W} / \mathrm{m}^{2}\right)$ & cond & conduction \\
\hline$R$ & radius $(\mathrm{mm})$ & $\operatorname{conv}$ & convection \\
\hline$r_{m}$ & $\begin{array}{l}\text { ratio of total surface area (pillars and } \\
\text { base) to footprint area }\end{array}$ & $d$ & device \\
\hline$T$ & temperature $\left({ }^{\circ} \mathrm{C}, \mathrm{K}\right)$ & evap & at completion of evaporation process \\
\hline$t$ & time $(\mathrm{s})$ & $E W$ & electrowetting \\
\hline$w$ & width (mm) & gen & generation in control volume \\
\hline$z$ & pillar height $(\mu \mathrm{m})$ & $\begin{array}{l}\text { in } \\
\text { latent }\end{array}$ & $\begin{array}{l}\text { into control volume } \\
\text { latent }\end{array}$ \\
\hline \multicolumn{2}{|l|}{ Symbols } & liq & liquid ribbon \\
\hline$\infty$ & ambient & out & out of control volume \\
\hline \multirow[t]{14}{*}{$\forall$} & volume & $\mathrm{rad}$ & radiation \\
\hline & & $s$ & stripe \\
\hline & & seg & circular segment \\
\hline & & sens & sensible \\
\hline & & $S i$ & silicon \\
\hline & & stored & stored in control volume \\
\hline & & $T i$ & titanium \\
\hline & & top & top surface of device \\
\hline & & $w$ & water \\
\hline & & 0 & initial \\
\hline & & \multicolumn{2}{|c|}{ Superscripts } \\
\hline & & 0 & previous time step \\
\hline & & 1 & current time step \\
\hline & & $*$ & Wenzel state \\
\hline
\end{tabular}




\section{Introduction}

The evaporation of droplets from a surface has diverse applications including coating [1], printing [2], cleaning [3], and two-phase thermal management [4]. As the mass transport from an evaporating droplet is directly related to its wettability on the surface [5], manipulation of droplet wettability by chemical [6], electrical [7], and other means [8,9] has been explored. The evaporation rate of a wetting droplet generally remains constant during the pinned-contact-line stage of evaporation [10]; a higher degree of wettability results in the capacity to support a higher evaporative transport rate.

The concept of using electrically actuated droplets as a thermal management technique has been investigated. Most prior work has focused on utilizing the sensible heating of droplets to remove heat from a surface [11-15]. In a typical configuration, an array of individually addressable electrodes moves a droplet to the desired location, where it absorbs heat and is subsequently removed and replaced by a cool droplet. Continuous, fast repetition of this process can maintain a cooling effect. Kumari and Garimella evaluated the heat transfer capacity of an electrowetting (EW)-based discrete droplet cooling approach [13]. They showed that the heat dissipation of such a system was comparable to a microchannel cooling system operating at the same pumping power.

Heat dissipation by phase change offers an attractive alternative to sensible heat-based thermal management. The evaporation of liquid droplets on heated surfaces has been the focus of numerical [16] and experimental [6,17-20] studies. Crafton and Black [19] investigated the evaporation of small water droplets with initial contact angles of up to $113^{\circ}$, and found that the evaporation rates were essentially constant through the lifetime of the drops and higher for drops with lower initial contact angles. Recently, Cheng and Chen [21] used EW actuation to deliver large (up to $25 \mu \mathrm{L}$ ) water droplets to a hot spot (initial surface temperature $\sim 180^{\circ} \mathrm{C}$ ) on a surface, with cooling being achieved by evaporation and nucleate boiling. Continuous hot spot cooling with a series of electrically actuated droplets was also demonstrated.

The present study seeks to further investigate the concept of evaporative cooling using small liquid volumes by characterizing the evaporation of narrow liquid ribbons from heated surfaces microstructured to create localized regions with different degrees of wettability. EW actuation causes the liquid ribbon to transition from a state of poor wetting to one of enhanced wetting. 
Infrared (IR) thermography is employed to characterize the cooling effect of the ribbons throughout the evaporation process, while a goniometer simultaneously monitors the ribbon shape. An energy balance analysis is used to estimate the heat transport in poor- and enhanced-wetting configurations. Conclusions drawn from this work help evaluate the prospects of using a phasechange-based EW device for site-specific thermal management.

\section{Experimental Setup and Procedures}

\subsection{Device Description}

The device is illustrated in Fig. 1(a). The base substrates are $150 \mu \mathrm{m}$ thick p-silicon wafers (University Wafer). Positive photoresist (AZ 9260, AZ Electronic Materials) is spin-coated on the wafer and patterned using standard photolithography techniques to serve as a protective mask for etching. Bosch-process deep reactive ion etching (ASE, Surface Technology Systems) anisotropically removes material from the exposed areas of the silicon wafer, resulting in the formation of high aspect ratio pillar arrays. The wafers are subjected to RCA cleaning [22], and a $9500 \AA$ layer of $\mathrm{SiO}_{2}$ is grown by wet oxidation to serve as the dielectric layer.

Table 1 lists the pillar height $z$, width $a$, and pitch $b$ of the surfaces tested along with the dimensionless roughness parameters $\phi$ and $r_{m} . \phi$ is the ratio of the total pillar tip surface area to the total footprint area of the substrate (i.e., $\phi=a^{2} / b^{2}$ ), and $r_{m}$ is the ratio of the total surface area (including sides of the pillars and base) to the total footprint area (i.e., $r_{m}=1+4 a z / b^{2}$ ). Each surface consists of two identical $4 \mathrm{~mm} \times 5 \mathrm{~mm}$ pillar-array regions separated by a $1 \mathrm{~mm}$ wide bare stripe (with no pillars). A thin, conformal coating of Teflon renders the surface hydrophobic; however, to achieve selective wetting of the bare stripe, it is masked with Kapton tape prior to the Teflon coating. A $30 \mathrm{~s}$ plasma clean $\left(\mathrm{O}_{2}\right.$ :Ar, 1:1 at $100 \mathrm{~W}, 1.5$ Torr $)$ removes the organic adhesives left by the Kapton tape after removal, while leaving the Teflon layer unaffected.

A $100 \mathrm{~nm}$ titanium layer is deposited on the backside of the device using an e-beam evaporator (SE 600, CHA Industries) at a deposition rate of $2 \AA / s$. Electrical heating of the titanium layer provides a constant heat flux to the device. The power input to the system is estimated from the measured current and voltage difference across the titanium layer. The uncertainty in these measurements is less than $1 \%$. Electrical connections to the titanium layer are 
made using electrically conductive silver epoxy (Duralco 120, Cotronics). Surfaces 1 and 2 are tested at $q_{T i}^{\prime \prime}=4710 \mathrm{~W} / \mathrm{m}^{2}$ which corresponds to an average device temperature in the absence of liquid of $80.1^{\circ} \mathrm{C}$; surfaces 3 and 4 are tested at $q_{T i}^{\prime \prime}=4330 \mathrm{~W} / \mathrm{m}^{2}$ which corresponds to an average device temperature in the absence of liquid of $80.5^{\circ} \mathrm{C}$.

\subsection{Working Fluid and Feeding Mechanism}

Deionized (DI), degassed water is used in the experiments. The contact angle of DI water on $\mathrm{SiO}_{2}$ and Teflon is measured to be $44^{\circ}$ and $115^{\circ}$, respectively, by a goniometer (CAM 100, KSV Instruments Ltd.). The confidence in all contact angle measurements is $\pm 1^{\circ}$. To achieve spontaneous wetting of the $\mathrm{SiO}_{2}$ stripe, a small amount $(0.01 \%$ by volume) of surfactant (Triton $\mathrm{X}-100$, Sigma-Aldrich) is added to the DI water to lower its surface tension, resulting in contact angles on $\mathrm{SiO}_{2}$ and Teflon of $20^{\circ}$ and $81^{\circ}$, respectively. When a small volume $(\sim 1 \mu \mathrm{L})$ of solution is deposited on the stripe, it spontaneously spreads to cover the stripe, remaining constrained by the chemical and physical phase boundaries. Since the contact line coincides with a boundary (i.e., where the pillar-array regions and bare stripe meet), the contact angle takes on an intermediate value bounded by the contact angles of the surrounding regions [23]. If the liquid volume is increased such that the contact angle of the surrounding phase is exceeded, the ribbon bulges outward at the middle of the stripe $[23,24]$. The contact line detaches from the boundary and advances until a new equilibrium shape is established. The critical volume for detachment from the boundary to occur was observed to be $\sim 1.5-2 \mu \mathrm{L}$ for the present configuration. Electrowetting of a liquid ribbon with volume $\sim 2 \mu \mathrm{L}$ yields a minor increase $(\sim 30 \%)$ in wetted area compared to its initial state; therefore, larger liquid volumes of 5 and $7 \mu \mathrm{L}$ are used in this work to facilitate a higher degree of wetted-area enhancement. This choice is discussed further in Section 3.1.

A syringe pump (PHD 2000, Harvard Apparatus) dispenses the prescribed volume at a rate of $1 \mu \mathrm{L} / \mathrm{s}$ through a syringe tip positioned just above the center of the hydrophilic $\mathrm{SiO}_{2}$ stripe. To explore the effects of EW-induced spreading on the heat transfer from the surface, experiments with and without EW are performed, and each test case is repeated three times. After the liquid is dispensed, a voltage bias of $400 \mathrm{~V}$ DC is applied for a duration of $1 \mathrm{~s}$ between the feeding syringe and an electrode contacting the base silicon substrate, causing the liquid ribbon to spread into the pillar arrays. The roughness elements provide pinning sites for the contact line, thus preventing the liquid from retracting immediately after the EW voltage is removed (as would occur on a 
smooth surface). The dynamics of the contact line will be discussed further in Section 3.1. In an EW system, Joule heating of the liquid volume is proportional to the square of the electric field. However, the present work uses a DC bias, and therefore, the liquid ribbon behaves as a perfect conductor and no electric field is present [25].

\subsection{Measurement Equipment and Techniques}

A schematic diagram of the test setup is shown in Fig. 2. The shape of the liquid ribbon along its axis is continually monitored using a goniometer and fiber-optic xenon lamp (XL 300-M, Henke-Sass Wolf $\mathrm{GmbH}$ ) that acts as a backlight. As discussed in Section 2.2, the liquid volumes studied in this work lead to a bulging ribbon shape; hence the widest cross-section of the ribbon is recorded during the evaporation process. The axial dimension of the ribbon remains constant (equal to the stripe length of $5 \mathrm{~mm}$ ) through all but the final moments before complete dry-out. The resolution of the goniometer is $11.1 \mu \mathrm{m} / \mathrm{pixel}$, and frames are recorded at $0.5 \mathrm{~s}$ intervals.

The titanium layer on the underside of the device is coated with a black paint (Krylon \#1602) of known emissivity of 0.95 [26]. An infrared (IR) camera (SC7500, FLIR Inc.) with attached mid-wavelength IR lens $(3.7-5.1 \mu \mathrm{m})$ records the temperature distribution of the painted surface of the device. The field of view of the lens is $14.4 \mathrm{~mm} \times 18.0 \mathrm{~mm}$, yielding a resolution of 56.25 $\mu \mathrm{m} /$ pixel. Images are captured at 100 frames per second (fps) and averaged at 20 fps (i.e., effectively recorded at $5 \mathrm{fps}$ ). Measurements were performed in a lab environment maintained at $21.5{ }^{\circ} \mathrm{C}$ and $29 \%$ relative humidity.

All image processing was performed with custom-written code in MATLAB [27]. The image processing procedure described in Chauvet et al. [28] is used to account for non-uniformity of the IR detector response and unwanted fluxes in the system, including those due to the Narcissus effect and non-focused radiation external and internal to the camera [29]. The camera is allowed to warm up for $\sim 2 \mathrm{hrs}$, beyond which period the camera temperature remains at a stable level. Prior to testing each surface, an image of the painted surface of the device is recorded at ambient temperature. Subtracting the ambient-temperature image from images obtained during heated test runs corrects for the effects of unwanted fluxes [28]. To correct for non-uniformity of the IR detector, the camera is calibrated using a high-sensitivity, high-stability blackbody (2000 series, SBIR Inc.). An ambient-temperature image of the blackbody surface is subtracted from subsequent images recorded at regular intervals throughout the range of interest $\left(21.5-95{ }^{\circ} \mathrm{C}\right)$. 
The IR detector signal is expressed in digital levels (DL); $12900 \mathrm{DL}$ span the $73.5^{\circ} \mathrm{C}$ range, thus the resolution is $175.5 \mathrm{DL} /{ }^{\circ} \mathrm{C}$. For each pixel, a fourth-order polynomial relates the blackbody temperature to the ambient-subtracted DL signal. In this manner, non-uniformity of the IR detector is mitigated. The maximum device temperature encountered in the present work is $81{ }^{\circ} \mathrm{C}$; at this level, the standard deviation of the pixel-by-pixel calibration procedure is $100 \mathrm{DL}\left(0.57^{\circ} \mathrm{C}\right)$, and the temperature measurement confidence is estimated to be $\pm 0.6{ }^{\circ} \mathrm{C}$.

\section{Results and Discussion}

\subsection{Temporal Evolution of Liquid Ribbon Shape}

As the liquid is being dispensed, it spontaneously spreads to cover the hydrophilic $\mathrm{SiO}_{2}$ stripe; as the ribbon volume is increased, a bulge forms at the axial midsection of the ribbon. The contact line of the ribbon advances through the pillar arrays until an equilibrium shape is reached. The degree of initial spreading is related to the roughness of the surface. Higher- $r_{m}$ surfaces exhibit a lower degree of initial spreading and a higher initial contact angle, which is consistent with hysteresis behavior in the Wenzel state [30]. The goniometer captures the temporal evolution of the widest cross-section of the ribbon (at the axial midpoint of the stripe as shown in Fig. 2). Table 2 presents the maximum wetted width $w$, apparent contact angle $\theta$, and total evaporation time $t_{\text {evap }}$ of the liquid ribbon for the tests conducted with and without electrowetting.

The wetting by a liquid in intimate contact with the roughness elements of a surface is described by Wenzel's equation [31]: $\cos \theta^{*}=r_{m} \cos \theta$ where $\theta^{*}$ and $\theta$ are the contact angles in the Wenzel state and on a smooth surface, respectively. The natural tendency of a surface with respect to wetting is amplified when roughness elements are added and the Wenzel state is realized - i.e., a smooth hydrophilic surface becomes more hydrophilic with the addition of roughness elements that are wetted by the liquid. In the present work, application of the EW voltage spreads the liquid ribbon into the pillar arrays surrounding the stripe. The liquid ribbon does not spread in the axial direction of the stripe (onto the flat Teflon region surrounding the active device area), as this would lead to a higher surface energy (manifest as a larger apparent contact angle). The shape of the electrowetted ribbon resembles that of an elongated droplet as the contact line remains 
pinned at the axial boundaries of the stripe (see Fig. 3). For highly spreading cases, $w$ can increase beyond the length of the stripe (e.g., $7 \mu \mathrm{L}$ ribbons under EW on Surfaces 3 and 4; see Table 2).

The evaporation modes observed for the liquid ribbons are essentially the same as those of evaporating droplets [10]. As the ribbon evaporates, there is initially a constant-width mode, where the contact line remains pinned and the contact angle decreases. When a critical contact angle is reached, the contact line depins and recedes, with the contact angle remaining constant as the ribbon width decreases. Finally, near the end of the ribbon lifetime, the width and contact angle rapidly decrease and complete dry-out occurs. The critical contact angle for the start of recession of the contact line was not resolved in this work, as the ribbon shape was not recorded at a frame rate high enough to capture the moment of depinning. The duration of the constant width mode and rate of contact line recession depend on the roughness of the surface. To illustrate this, Fig. 4 presents the width of the ribbon $w$ as a function of time. For the cases with no EW actuation, the duration of the constant-width mode is longer for surfaces with higher $r_{m}$, with Surface 3 resisting the depinning of the contact line the longest. In cases with EW actuation, the duration of the constant-width mode is reduced for all cases because $\theta_{E W}$ is closer to the critical contact angle for detachment than $\theta_{0}$. In terms of spreading enhancement, the increase in $w$ is greatest for the surfaces with higher $r_{m}$ as shown in Fig. 4. Surface 3 exhibits the most significant width enhancement, with $w_{E W} / w_{0}$ of 3.0 and 2.8 for the 5 and $7 \mu \mathrm{L}$ ribbons, respectively; in contrast, Surface 2 yields enhancements of less than $50 \%$.

\subsection{Temporal Evolution of Device Temperature}

The liquid ribbon cross-sectional shape and the device temperature distribution for Surface 3 are presented as a function of time in Fig. 5. The device is less than $150 \mu \mathrm{m}$ thick and has relatively high thermal conductivity $\left(k_{S i}=149 \mathrm{~W} / \mathrm{m} \mathrm{K}\right)$; therefore temperature gradients through the thickness of the device are neglected. The cooling effect of the liquid is not limited to the wetted area of the ribbon, but rather the liquid acts as a sink, drawing heat from the surrounding areas of the device and creating a temperature suppression centered at the location of the liquid ribbon. Similar temperature profiles were observed in a drop-wise cooling study in Ref. [20]. Maximum device temperature drops in the present work were measured to be $4.8-9.0^{\circ} \mathrm{C}$, and typically occurred at a time between $80-90 \%$ of $t_{\text {evap }}$, which is consistent with Ref. [20]. Surfaces 2 and 3 yielded the minimum and maximum cooling effects, respectively. On Surface 3, 
maximum temperature drops for a ribbon volume of 5 (7) $\mu \mathrm{L}$ were 5.5 (7.4) and $7.9(9.0)^{\circ} \mathrm{C}$, without and with EW, respectively. The lower device temperature of the EW case results from the increased surface area, which allows for a higher evaporation rate and thus a higher heat transfer rate, albeit for a shorter duration. The temperature drops observed in this work were much lower than those of Ref. [21] because the initial surface temperature in this work $\left(\sim 80^{\circ} \mathrm{C}\right)$ does not facilitate rapid evaporation/boiling of the liquid unlike the case in [21], which had an initial surface temperature of $\sim 180^{\circ} \mathrm{C}$.

\subsection{Transient Heat Transfer Analysis}

\subsubsection{Heat balance formulation}

The thermal performance of the device may be assessed by performing a heat balance on the control volume (CV) shown in Fig. 6. The CV includes the silicon substrate of the device directly under the liquid ribbon. The length of the $\mathrm{CV}$ is constant and equal to the stripe length $l_{s}$, while its width varies with time and is equal to the ribbon width $w$ (see Section 3.3.2 for details of the implicit time-stepping scheme used). The heat balance on the CV is

$$
q_{\text {in }}-q_{\text {out }}+q_{\text {gen }}=q_{\text {stored }}
$$

There is no heat generation term, as the CV does not include the titanium layer. The storage term accounts for the transient temperature change of the silicon substrate:

$$
q_{\text {stored }}=\rho_{S i} w l_{s} \delta c_{p, S i} \frac{\partial T}{\partial t}
$$

where $\rho, c_{p}$, and $\delta$ are the density, specific heat, and thickness of the substrate.

Conduction in the substrate through the vertical walls of the CV $\left(q_{\text {cond }}\right)$ and electrical heating of the titanium layer $\left(q_{T i}\right)$ contribute to $q_{\text {in }}$ as

$$
\begin{aligned}
& q_{c o n d}=-k_{S i} \vec{A} \cdot \nabla T \\
& q_{T i}=q_{T i}^{\prime \prime} w l_{s}
\end{aligned}
$$

where $k$ is the thermal conductivity, $\vec{A}$ is the area vector of the CV face, and $\nabla T$ is the temperature gradient at the $\mathrm{CV}$ face. The heat input from the titanium layer is proportional to the area of the horizontal CV face $w l_{s}$. 
Natural convection $q_{c o n v}$ and radiation $q_{\text {rad }}$ from the top and bottom surfaces contribute to $q_{o u t}$, as does the heat lost to the liquid ribbon $q_{\text {liq. }}$. Radiation from the exposed regions of the top surface is not considered, as the emissivity $\varepsilon$ of polished silicon in the 3.7-5.1 $\mu \mathrm{m}$ wavelength is $<0.1$ [32]. Radiation from the bottom surface is given by

$$
q_{\text {rad }, b o t}=\varepsilon \sigma w l_{s}\left(T^{4}-T_{\infty}^{4}\right)
$$

where the device temperature $T$ and ambient temperature $T_{\infty}$ are in Kelvin and the emissivity $\varepsilon$ of the black painted surface is 0.95 [26]. To determine the natural convection coefficients on the top and bottom of the device, an energy balance on the $\mathrm{CV}$ in the absence of a liquid ribbon is performed. In this case, the temperature of the device is essentially uniform spatially (thus $q_{\text {cond }}=$ $0)$ and temporally $(\partial T / \partial t=0)$. The heat input from the titanium layer must balance the losses due to convection and radiation:

$$
q_{T i}^{\prime \prime} w l_{s}=h_{b o t} w l_{s}\left(T-T_{\infty}\right)+h_{t o p} w l_{s}\left(T-T_{\infty}\right)+\varepsilon \sigma w l_{s}\left(T^{4}-T_{\infty}^{4}\right)
$$

Taking representative values from the experiment of $q_{T i}^{\prime \prime}=4710 \mathrm{~W} / \mathrm{m}^{2}, T=80^{\circ} \mathrm{C}(353 \mathrm{~K}), T_{\infty}=$ $21.5^{\circ} \mathrm{C}(295 \mathrm{~K})$, and assuming that $h_{t o p}=2 \times h_{b o t}$ as in correlations suggested by McAdams [33], $h_{b o t}$ is estimated to be $\sim 25 \mathrm{~W} / \mathrm{m} \mathrm{K}$.

\subsubsection{Liquid ribbon heat dissipation}

Data reduction was performed in MATLAB [27]. Eq. (1) is discretized using an implicit time-stepping scheme as

$$
\begin{aligned}
& \sum_{\text {faces }}-k_{S i} \vec{A}^{(1)} \cdot \nabla T^{(1)}+q_{T i}^{\prime \prime} w^{(1)} l_{s}-q_{\text {liq }}-h_{\text {top }} A_{\text {top }}^{(1)}\left(T^{(1)}-T_{\infty}\right)-h_{b o t} w^{(1)} l_{s}\left(T^{(1)}-T_{\infty}\right) \\
& -\varepsilon \sigma w^{(1)} l_{s}\left(T^{(1) 4}-T_{\infty}^{4}\right)=\rho_{S i} w^{(1)} l_{s} \delta c_{p, S i} \frac{T^{(1)}-T^{(0)}}{t^{(1)}-t^{(0)}}
\end{aligned}
$$

where the superscripts 1 and 0 indicate that parameters are evaluated at the current time step and previous time step, respectively. With the implicit formulation employed, there is no connection between the volume of the $\mathrm{CV}$ at one instant and the next. Since $w$ decreases during the evaporation process, the exposed surface area of the device top $A_{\text {top }}$ must be calculated at each time step. Assuming the wetted area under the ribbon to take the shape of a circular segment between the axial stripe boundaries and the widest extent of the ribbon (see Fig. 7), $A_{\text {top }}$ may be estimated as 


$$
A_{t o p}=w l_{s}-\left(2 A_{s e g}+w_{s} l_{s}\right)
$$

where

$$
A_{\text {seg }}=\frac{R^{2}}{2}(\alpha-\sin \alpha)
$$

Using the relations in Fig. 7, $H=\left(w-w_{s}\right) / 2, C=l_{s}$, and the circle radius $R$ and central angle $\alpha$ are given by

$$
\begin{aligned}
& R=\frac{H}{2}+\frac{C^{2}}{8 H} \\
& \alpha=2 \arccos \frac{R-H}{R}
\end{aligned}
$$

With the temperature distribution of the device known, the conductive heat flow into the $\mathrm{CV}$ (first term on the left hand side in Eq. (7)) is found by numerically calculating the gradient at each face. The convection, radiation, and storage terms are calculated at each pixel for a given time step. In this manner, $q_{\text {liq }}$ is determined as a function of time for the duration of the experiment.

The energy absorbed by the liquid ribbon from the device is related to the latent and specific heats of the liquid. Evaporation from the ribbon causes the absorption of energy in the amount of $\rho_{w} \forall h_{f g, w}$ where $h_{f g}$ is the latent heat of the liquid. Sensible heating of the liquid ribbon requires energy equal to $\rho_{w} \forall c_{p, w} \Delta T$ where $\Delta T$ is the temperature rise of the liquid. Ribbons of equal volume will absorb the same amount of latent energy to fully evaporate. The sensible heat required depends on the internal temperature rise of the liquid. In the present work, the internal temperature of the liquid ribbon is not monitored; however, taking water properties at $52^{\circ} \mathrm{C}(\rho=$ $987 \mathrm{~kg} / \mathrm{m}^{3}, h_{f g}=2378 \mathrm{~kJ} / \mathrm{kg}, c_{p}=4.182 \mathrm{~kJ} / \mathrm{kg} \mathrm{K}$ ) and assuming a temperature rise from ambient $\left(21.5^{\circ} \mathrm{C}\right)$ to the average device temperature observed under the liquid $\left(\sim 75^{\circ} \mathrm{C}\right)$, the relative magnitudes of latent and sensible energy may be compared. At a volume of 5 (7) $\mu \mathrm{L}$, the latent and sensible energies are 11.7 (16.4) and 1.1 (2.2) J, respectively. The values for sensible energy represent the maximum expected, and correspond to the entire volume of liquid being heated to $75^{\circ} \mathrm{C}$ before it evaporates away. The lower limit to sensible heating $(\sim 0 \mathrm{~J})$ corresponds to a process where the liquid ribbon experiences a negligible rise in its internal temperature (such as in the case of evaporation on an unheated substrate). At either limit of sensible heat gain, the energy 
dissipation due to the liquid ribbon is clearly dominated by latent heat absorption; thus a configuration that supports a higher evaporation rate is advantageous from a heat transfer standpoint.

Integration of the heat flow to the liquid ribbon over the duration of the ribbon lifetime yields the energy dissipation due to the ribbon

$$
E_{\text {liq }}=\int_{t_{0}}^{t_{\text {evap }}} q_{\text {liq }} d t
$$

This quantity should equal the sum of the latent and sensible heat energy of the ribbon, and, as discussed in the previous paragraph, it is predicted to be within the range of $11.7-12.8$ (16.4 18.6) $\mathrm{J}$ for 5 (7) $\mu \mathrm{L}$ ribbons. Fig. 8 shows the experimentally estimated cumulative energy dissipation of the 5 and $7 \mu \mathrm{L}$ volume ribbons on Surface 3 as a function of time, with and without EW. $E_{l i q}$ is estimated to be 12.9 (18.1) and 12.9 (19.9) $\mathrm{J}$ for 5 (7) $\mu \mathrm{L}$ ribbons without and with EW, respectively, which is within $\sim 10 \%$ of the expected range (a typical discrepancy for other runs, as well). Since the duration of EW runs is shorter than the runs without EW, the energy vs. time slopes are higher, indicating a higher rate of heat transfer. The slopes of non-EW runs are essentially constant throughout the entire evaporation process as the contact line remains pinned through nearly the entire lifetime of the liquid ribbon. This is consistent with the constant evaporation rate of a droplet in the pinned-diameter evaporation mode $[10,19]$.

Figure 9 shows the estimated heat flux and wetted area for $5 \mu \mathrm{L}$ liquid ribbons on Surface 3 as a function of time. Based on the initial width of these ribbons, the hydraulic diameter $(4 A / P$, where $P$ is the wetted perimeter of the ribbon) is 2.1 and $4.5 \mathrm{~mm}$ for cases without and with EW, respectively. The heat fluxes are observed to be $\sim 20 \mathrm{~kW} / \mathrm{m}^{2}$, which compares well with values reported in Ref. [17] for $\sim 4 \mathrm{~mm}$-diameter water droplets on surfaces heated to between 75 and $85^{\circ}$ C. As a result of constant evaporation rate, the non-EW run shows relatively constant heat flow of $q_{\text {liq }} \sim 0.2 \mathrm{~W}$ while the wetted area remains constant. For the EW case, the evaporation rate (and

$\left.q_{l i q}\right)$ is at a maximum immediately after the EW-induced spreading, and declines as the wetted area decreases. Continuous feeding of the ribbon at a rate matched to the evaporative mass loss would sustain the higher heat transfer rate indefinitely.

\subsubsection{Estimation of effective liquid ribbon convection coefficient}


The effective convective coefficient on the device face under the liquid ribbon at a given time may be estimated as

$$
h_{\text {avg }}=q_{\text {liq }} / A_{\text {liq }}\left(T_{d, a v g}-T_{\text {liq }}\right)
$$

where $T_{d, a v g}$ is the average device temperature under the ribbon and $T_{\text {liq }}$ is the bulk temperature of the liquid. Since the internal temperature of the liquid is not measured, the temperature to use for $T_{l i q}$ is unclear; however, a value for $h_{a v g}$ may be estimated by the analysis that follows.

The evaporative flux from the liquid ribbon is essentially constant with time; thus the ribbon volume is assumed to vary linearly over the duration of the evaporation process such that at time $t$, the volume is

$$
\forall=\forall_{0}-\dot{m} t
$$

where $\dot{m}=\rho_{w} \forall_{0} / t_{\text {evap }} \cdot{ }^{1}$ Noting that the slope of $E_{\text {liq }}$ vs. time is essentially constant (see Fig. 8), $q_{\text {sens }}$ is assumed to be constant and described by

$$
q_{\text {sens }}=\rho_{w} c_{p, w} \forall \frac{d T_{l i q}}{d t}=\beta
$$

where $\beta$ is a constant value to be determined. Solving the differential equation with the initial and final conditions $T_{\text {liq }}(t=0)=T_{\infty}$ and $T_{\text {liq }}\left(t \rightarrow t_{\text {evap }}\right)=T_{d, \text { avg }}\left(t_{\text {evap }}\right)$ yields an expression for bulk liquid temperature

$$
T_{\text {liq }}=T_{0}+\frac{\beta}{c_{p, w} \dot{m}} \ln \left(\frac{\forall_{0}}{\forall_{0}-\dot{m} t / \rho_{w}}\right)
$$

With the variables on the right hand side of Eq. (13) thus known, $h_{\text {avg }}$ may be estimated. Fig. 10 presents the typical relationship between $T_{l i q}$ and $h_{\text {avg }}$, as estimated using the assumptions described above. During the evaporation process, the average device temperature $T_{d \text {,avg }}$ remains relatively constant at $\sim 75^{\circ} \mathrm{C}-$ a cooling effect of $\sim 5.5^{\circ} \mathrm{C}$. The value of $h_{\text {avg }}$ is found to be in the range of $400-600 \mathrm{~W} / \mathrm{m}^{2} \mathrm{~K}$ over the majority of the liquid ribbon lifetime; it rises rapidly as

\footnotetext{
${ }^{1}$ Backing out the sensible heating of the ribbon using $q_{\text {sens }}=q_{\text {liq }}-q_{\text {latent }}$ would be preferred; however, as noted in the previous section, the total $E_{l i q}$ estimated by the procedure described slightly overpredicts the maximum expected value. Since $q_{\text {sens }}$ is very small compared to $q_{\text {latent }}$, this approach does not provide the required resolution.
} 
$t \rightarrow t_{\text {evap }}$ in response to $\left(T_{d, \text { avg }}-T_{\text {liq }}\right)$ approaching 0 . While this analysis is approximate, it allows an estimation of the effective convection coefficient. A more accurate calculation of $h_{\text {avg }}$ could be obtained if the temporal variation of the bulk temperature as well as the wetted footprint of the liquid ribbon as it evaporates were obtained by measurement (as opposed to the estimation described in Section 3.3.2).

\subsection{Practical Implementation}

The device described in this work is capable of dissipating moderate heat fluxes on the order of $20 \mathrm{~kW} / \mathrm{m}^{2}$ under the conditions of the experiment. The use of electrowetting facilitates control of the surface area that is cooled by the liquid ribbon. Discrete droplet systems, such as those described by Kumari and Garimella [13] and others [12, 15], have the capacity to dissipate heat fluxes an order of magnitude higher by implementing high frequency actuation of droplets across a heated surface; the device described in the present work is suited for applications where moderate heat dissipation is required over an area that varies temporally. Latent energy-based based systems can reach this level of heat dissipation, but must rely on suitably high surface temperatures to facilitate nucleate boiling as in Ref. [21], or use drops of small diameter: Crafton and Black [19] dissipated heat fluxes of over $130 \mathrm{~kW} / \mathrm{m}^{2}$ with heating conditions similar to the present work using droplets under $1 \mathrm{~mm}$ in diameter. They showed that heat flux decreases with increase in droplet diameter; the largest water droplet they used was $1.35 \mathrm{~mm}$ in diameter and it yielded a heat flux of $90 \mathrm{~kW} / \mathrm{m}^{2}$. By reducing the ribbon volume and/or increasing the wall temperature, the system described in the present work would be able to achieve improved thermal performance.

Similar to the other EW-based thermal solutions proposed in the literature, the device in this work would readily lend itself to feeding by an array of individually addressable electrodes or conventional pumping, which would serve to maintain the heat dissipation at a steady, high level. One possible device implementation is depicted in Fig. 11 where liquid is transported via individually addressable electrodes on a cover plate. When the desired location is reached, droplets are spread on the device by activating the electrode on the surface. Another possibility is to feed a hydrophilic stripe by capillary action from a reservoir [34], eliminating the need for active pumping. The stripe could be forced to spread at particular locations by activating individually addressable electrodes. An array of such stripes could cool a large surface area, and 
utilizing sub- $\mu \mathrm{L}$ liquid volumes would improve thermal performance. Future work will focus on more practical device designs implementing low thermal conductivity substrates and co-planar electrodes [35].

\section{Conclusions}

An electrowetting-based device that utilizes chemical and structural patterning to facilitate the selective wetting of water into ribbon shapes is investigated. The liquid ribbons were subject to a constant heat flux condition. Various surface roughness patterns and liquid volumes were tested, and the influence of electrowetting-induced spreading on the wettability and thermal performance of the ribbons was explored. Electrically actuated ribbons exhibited significant increases in wetted surface area (up to 170\%) and were able to sustain higher evaporation rates than ribbons without actuation (increases up to 57\%). Under the conditions of the experiment, heat fluxes of $\sim 20$ $\mathrm{kW} / \mathrm{m}^{2}$ were dissipated by the liquid ribbons. The performance of devices based on this concept could be improved by using smaller liquid volumes and/or higher surface temperatures.

\section{Acknowledgement}

The authors acknowledge financial support for this work from the Cooling Technologies Research Center, a National Science Foundation Industry/University Cooperative Research Center at Purdue University. CPM was supported by the U.S. Department of Defense through the SMART program. CPM thanks Brendan Hanrahan for assistance in developing the device

fabrication process and Tim Persoons for sharing the image processing code. Hemanth Dhavaleswarapu originally conceptualized the device explored in this work.

\section{References}

1 L. Xu, H. Zhu, H.E. Ozkan, H.W. Thistle, Evaporation rate and development of wetted area of water droplets with and without surfactant at different locations on waxy leaf surfaces, Biosyst. Eng. 106 (2010) 58-67. 
2 J. Fukai, H. Ishizuka, Y. Sakai, M. Kaneda, M. Morita, A. Takahara, Effects of droplet size and solute concentration on drying process of polymer solution droplets deposited on homogeneous surfaces, Int. J. Heat Mass Transfer 49 (2006) 3561-3567.

3 B. Bhushan, K. Koch, Y.C. Jung, Biomimetic hierarchical structure for self-cleaning, Appl. Phys. Lett. 93 (2008) 093101 (3 pp.)

4 W.M. Grissom, F.A. Wierum, Liquid spray cooling of a heated surface, Int. J. Heat Mass Transfer 24 (1981) 261-271.

5 K.S. Birdi, D.T. Vu, Wettability and the evaporation rates of fluids from solid surfaces, J. Adhes. Sci. Technol. 7 (1993) 485-493.

6 S. Chandra, M. di Marzo, Y.M. Qiao, P. Tartarini, Effect of liquid-solid contact angle on droplet evaporation, Fire Saf. J. 27 (1996) 141-158.

7 C. Quilliet, B. Berge, Electrowetting: a recent outbreak, Curr. Opin. Colloid Interface Sci. 6 (2001) 34-39.

8 Y. Zimmels, Thermodynamics of wetting of solid surface by magnetizable fluids, J. Magn. Magn. Mater. 122 (1993) 241-247.

9 A.A. Darhuber, S.M. Troian, Principles of microfluidic actuation by modulation of surface stresses, Annu. Rev. Fluid Mech. 37 (2005) 425-455.

10 H. Hu, R.G. Larson, Evaporation of a sessile droplet on a substrate, J. Phys. Chem. B 106 (2002) 1334-1344.

11 V. Bahadur, S.V. Garimella, Energy minimization-based analysis of electrowetting for microelectronics cooling applications, Microelectron. J. 39 (2008) 957-965.

12 P.Y. Paik, V.K. Pamula, K. Chakrabarty, Adaptive cooling of integrated circuits using digital microfluidics, IEEE Trans. Very Large Scale Integr. VLSI Syst. 16 (2008) 432-443. 
13 N. Kumari, S.V. Garimella, Characterization of the heat transfer accompanying electrowetting or gravity-induced droplet motion, Int. J. Heat Mass Transfer 54 (2011) 4037-4050.

14 E. Baird, K. Mohseni, Digitized heat transfer: A new paradigm for thermal management of compact micro systems, IEEE Trans. Compon. Pack. Technol. 31 (2008) 143-151.

15 H. Oprins, J. Danneels, B. Van Ham, B. Vandevelde, M. Baelmans, Convection heat transfer in electrostatic actuated liquid droplets for electronics cooling, Microelectron. J. 39 (2008) 966-974. 16 O.E. Ruiz, W.Z. Black, Evaporation of water droplets placed on a heated horizontal surface, J. Heat Transfer - Trans. ASME 124 (2002) 854-863.

17 M. di Marzo, D.D. Evans, Evaporation of a water droplet deposited on a hot high thermalconductivity surface, J. Heat Transfer - Trans. ASME 111 (1989) 210-213.

18 M. di Marzo, P. Tartarini, Y. Liao, D. Evans, H. Baum, Evaporative cooling due to a gently deposited droplet, Int. J. Heat Mass Transfer 36 (1993) 4133-4139.

19 E.F. Crafton, W.Z. Black, Heat transfer and evaporation rates of small liquid droplets on heated horizontal surfaces, Int. J. Heat Mass Transfer 47 (2004) 1187-1200.

20 L. Tarozzi, A. Muscio, P. Tartarini, Experimental tests of dropwise cooling on infraredtransparent media, Exp. Thermal Fluid Sci. 31 (2007) 857-865.

21 J.-T. Cheng, C.-L. Chen, Adaptive chip cooling using electrowetting on coplanar control electrodes, Nanoscale Microscale Thermophys. Eng. 14 (2010) 63-74.

22 M.J. Madou, Fundamentals of Microfabrication: The Science of Miniaturization, $2^{\text {nd }}$ ed., CRC Press, New York, 2002, pp. 11-12.

23 P. Lenz, R. Lipowsky, Morphological transitions of wetting layers on structured surfaces, Phys. Rev. Lett. 80 (1998) 1920-1923. 
24 M. Brinkmann, R. Lipowsky, Wetting morphologies on substrates with striped surface domains, J. Appl. Phys. 92 (2002) 4296-4306.

25 J.S. Hong, S.H. Do, K.H. Kang, I.S. Kang, A numerical investigation on AC electrowetting of a droplet, Microfluid. Nanofluid. 5 (2008) 263-271.

26 NASA Jet Propulsion Laboratory Web Site, last accessed 15 Dec. 2011. URL: <http://masterweb.jpl.nasa.gov/reference/paints.htm>

27 MATLAB, The Language of Technical Computing, Version 7.6, The Mathworks, Inc., Natick, MA, 2008.

28 F. Chauvet, S. Cazin, P. Duru, M. Prat, Use of infrared thermography for the study of evaporation in a square capillary tube, Int. J. Heat Mass Transfer 53 (2010) 1808-1818.

29 N. Horny, FPA camera standardization, Infrared Phys. Technol. 44 (2003) 109-119.

30 K.-Y. Yeh, L.J. Chen, Contact angle hysteresis on regular pillar-like hydrophobic surfaces, Langmuir 24 (2008) 245-251.

31 T.N. Wenzel, Surface roughness and contact angle, J. Phys. Colloid Chem. 53 (1949) 14661467.

32 N.M. Ravindra, K. Ravindra, S. Mahendra, B. Sopori, A.T. Fiory, Modeling and simulation of emissivity of silicon-related materials and structures, J. Electron. Mater. 32 (2003) 1052-1058.

33 W.H. McAdams, Heat Transmission, $3^{\text {rd }}$ ed., New York, McGraw-Hill, 1954.

34 A. Darhuber, J.M. Davis, S.M. Troian, Thermocapillary actuation of liquid flow on chemically patterned surfaces, Phys. Fluids 15 (2003) 1295-1304.

35 U.-C. Yi, C.-J. Kim, Characterization of electrowetting actuation on addressable single-side coplanar electrodes, J. Micromech. Microeng. 16 (2006) 2053-2059. 
Table 1. Pillar geometry and roughness parameters of the surfaces tested.

\begin{tabular}{ccc}
\hline \hline Surface & $\begin{array}{c}z(\mu \mathrm{m}), a \\
(\mu \mathrm{m}), b(\mu \mathrm{m})\end{array}$ & $\phi, r_{m}$ \\
\hline 1 & $5.25,20,28$ & $0.51,1.54$ \\
2 & $5.25,20,62$ & $0.10,1.11$ \\
3 & $21.1,20,28$ & $0.51,3.15$ \\
4 & $21.1,20,62$ & $0.10,1.44$ \\
\hline
\end{tabular}


Table 2. Width and apparent contact angle for initial and electrowetted states of 5 and $7 \mu \mathrm{L}$ liquid ribbons on the surfaces tested as measured at the widest cross-section of the liquid ribbon. The average of three runs is reported; the standard deviation for the parameters is $\sim 6 \%$ within each set of runs.

\begin{tabular}{ccccccc}
\hline \hline \multirow{2}{*}{ Surface } & \multicolumn{5}{c}{$5 \mu \mathrm{L}$} & \multicolumn{3}{c}{$7 \mu \mathrm{L}$} \\
\cline { 2 - 7 } & $\begin{array}{c}w_{0}, w_{E W} \\
(\mathrm{~mm})\end{array}$ & $\theta_{0}, \theta_{E W}\left({ }^{\circ}\right)$ & $t, t_{E W}(\mathrm{~s})$ & $\begin{array}{c}w_{0}, w_{E W} \\
(\mathrm{~mm})\end{array}$ & $\theta_{0}, \theta_{E W}\left({ }^{\circ}\right)$ & $t, t_{E W}(\mathrm{~s})$ \\
\hline 1 & $1.65,2.85$ & 97,43 & $68.8,56.3$ & $2.08,4.16$ & 101,36 & $84.2,81.8$ \\
2 & $2.01,3.11$ & 88,39 & $68.7,71.0$ & $2.73,3.92$ & 78,36 & $100.7,98.0$ \\
3 & $1.37,4.11$ & 117,30 & $69.1,43.9$ & $1.86,5.28$ & 119,24 & $89.9,57.4$ \\
4 & $1.70,4.35$ & 104,29 & $69.0,58.7$ & $1.87,5.23$ & 108,23 & $91.1,70.6$ \\
\hline
\end{tabular}




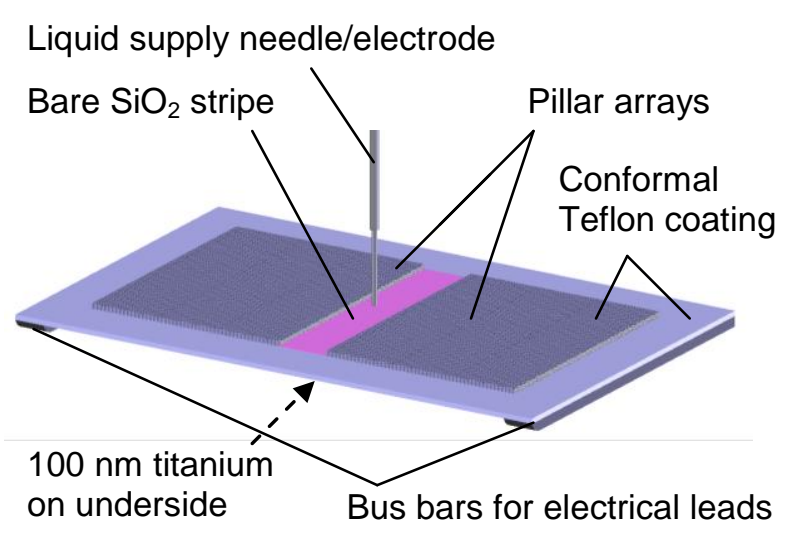

(a)

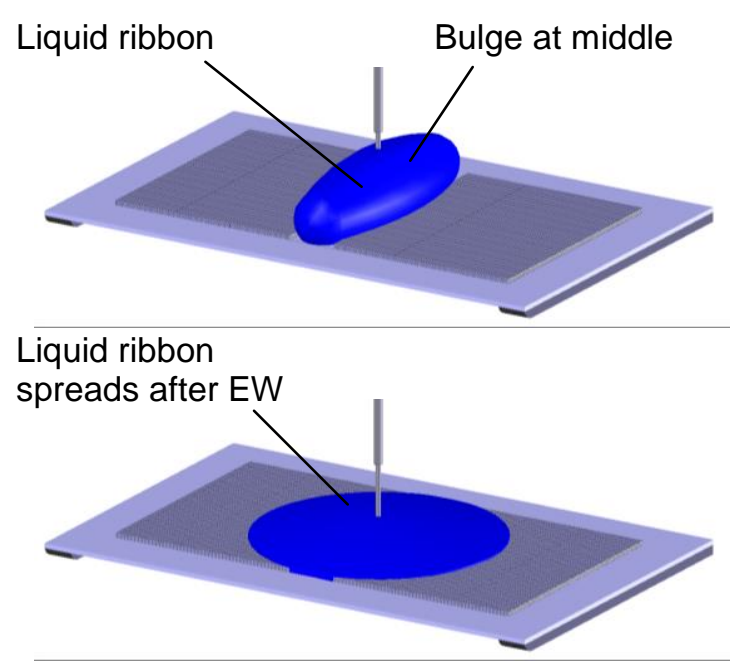

(b)

Fig. 1 (Color online) (a) Schematic illustration of the device and feeding mechanism. The entire topside of the device, with the exception of the $\mathrm{SiO}_{2}$ stripe, has a conformal layer of Teflon to promote selective wetting of the stripe. Electrical heating of the titanium layer on the device backside provides a constant heat flux. (b) A syringe pump dispenses the prescribed volume of liquid to the device where it spreads along the $\mathrm{SiO}_{2}$ stripe forming a liquid ribbon. Applying a voltage bias between the needle electrode and the silicon base substrate spreads the liquid through the pillar arrays. 


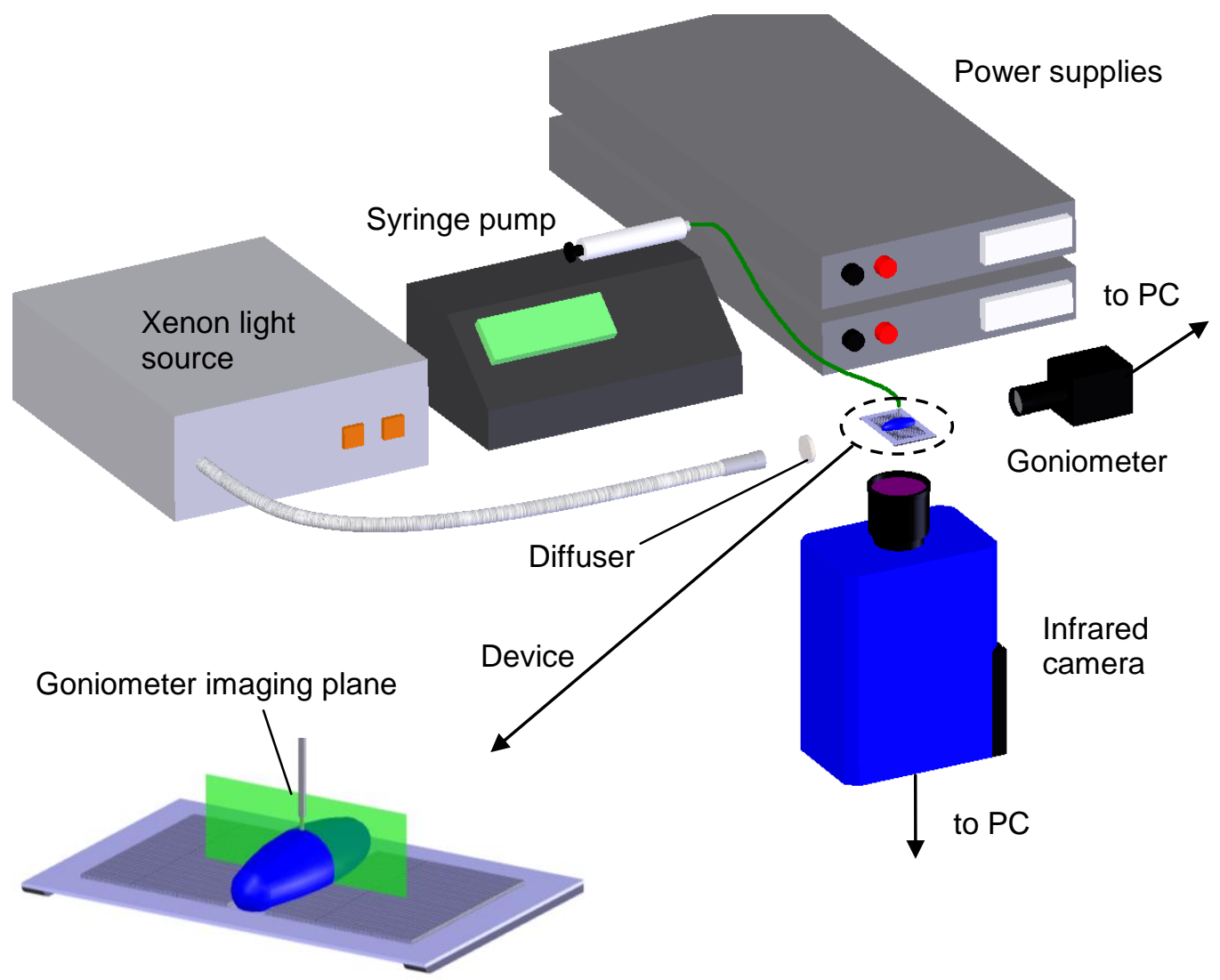

Fig. 2 (Color online) Perspective view of test setup. Electrical leads and linear stages are omitted for clarity. The goniometer monitors the shape of the liquid ribbon, while the infrared camera records the temperature distribution of the device backside. 


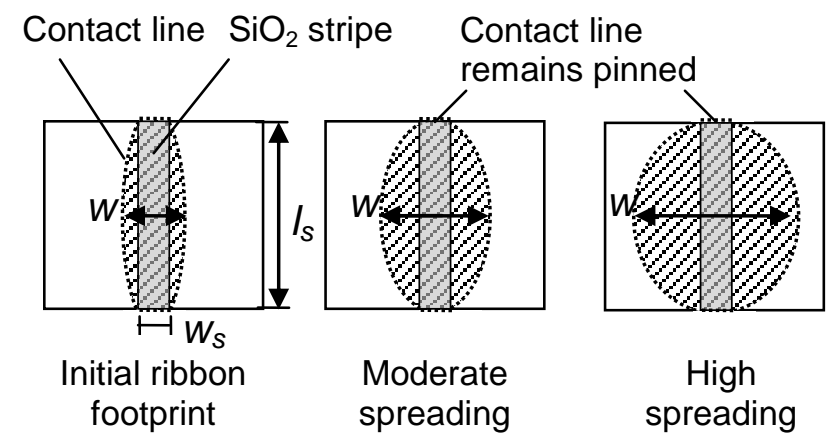

Fig. 3 (Color online) Plan view illustration of device showing liquid ribbon footprint under different conditions. Under EW actuation, the ribbon spreads through the pillar arrays, remaining pinned at the axial boundaries of the stripe. The footprint of moderately spreading cases (e.g., on Surface 2) resembles an elongated droplet, while the footprint of highly spreading cases resembles a circular droplet (e.g., on Surface 3). 


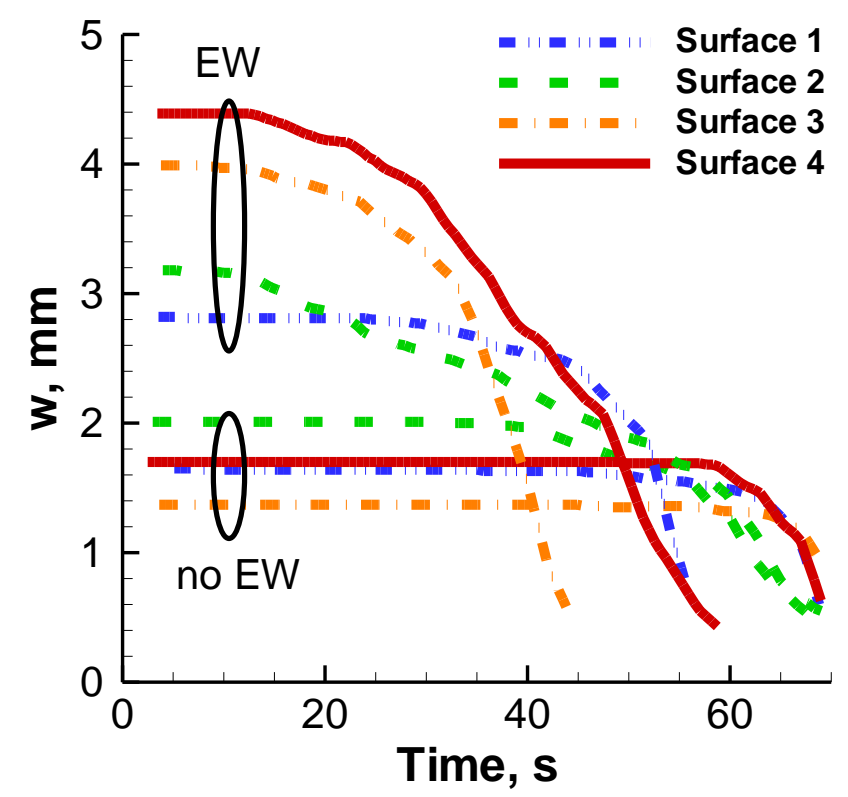

(a)

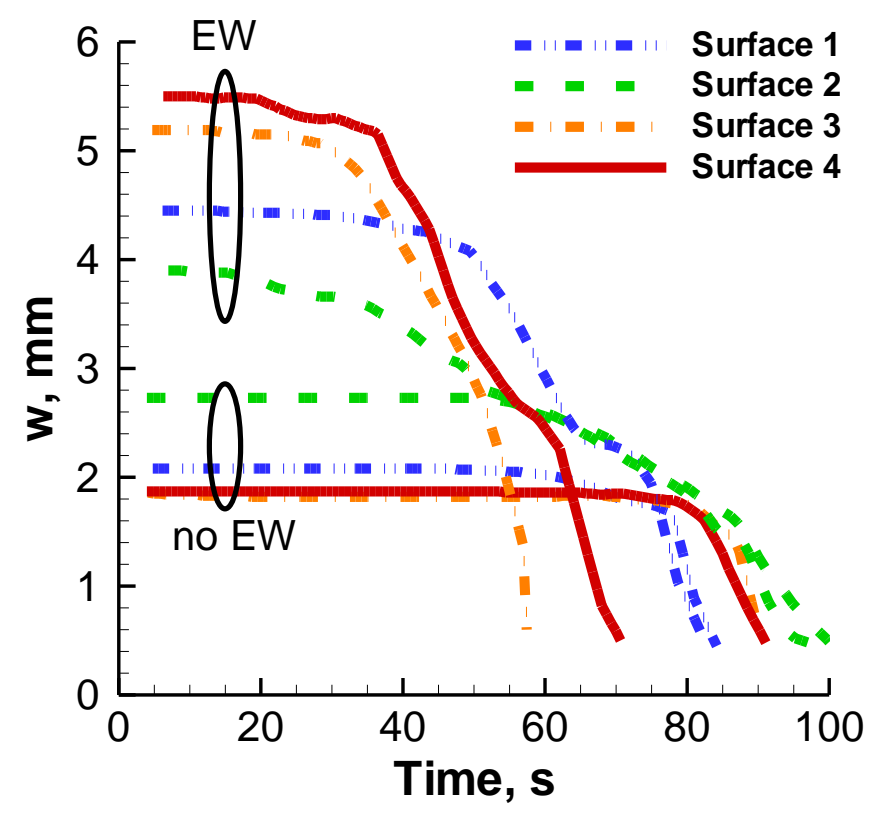

(b)

Fig. 4 (Color online) Width at stripe midsection $w$ as a function of time for (a) $5 \mu \mathrm{L}$ and (b) $7 \mu \mathrm{L}$ liquid ribbons. EW actuation spreads the ribbon, increasing $w$ and reducing $t_{\text {evap }}$ which indicates an enhanced evaporation rate. Surfaces 3 and 4 exhibit the largest change in these parameters. 


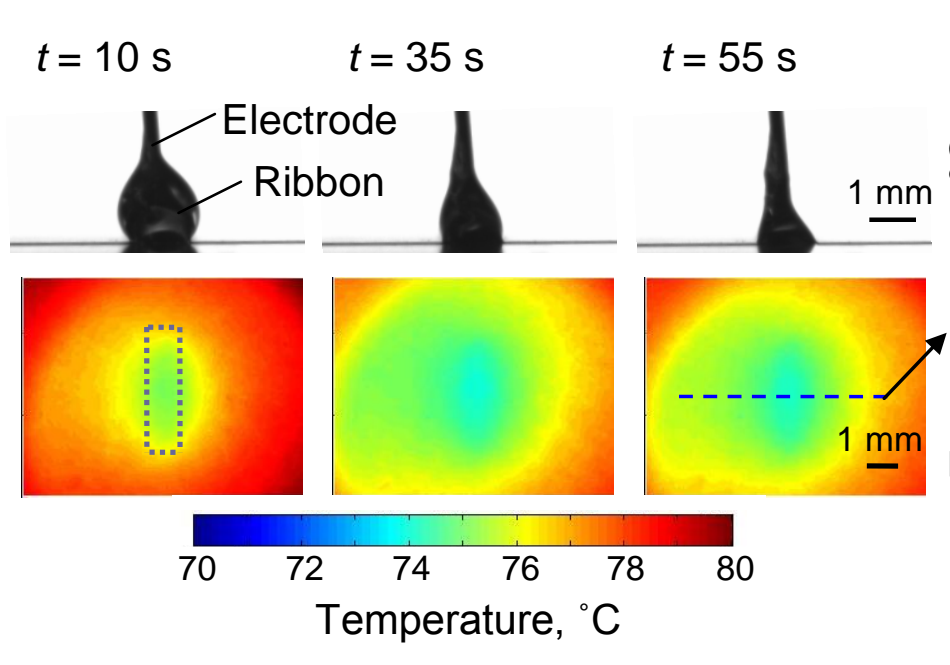

(a)

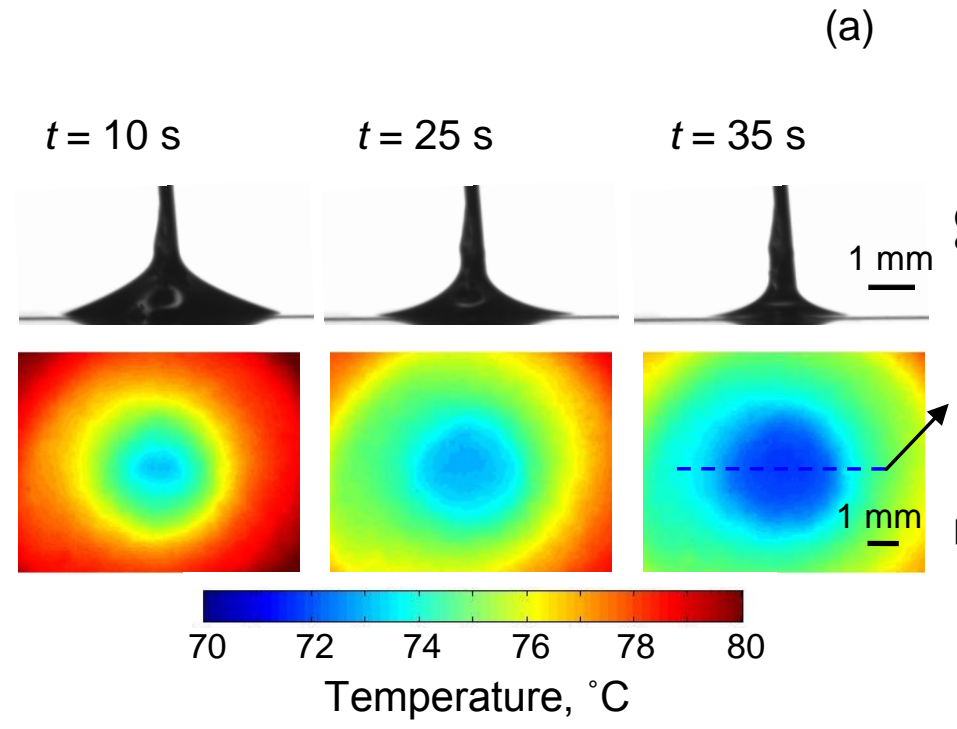

(b)
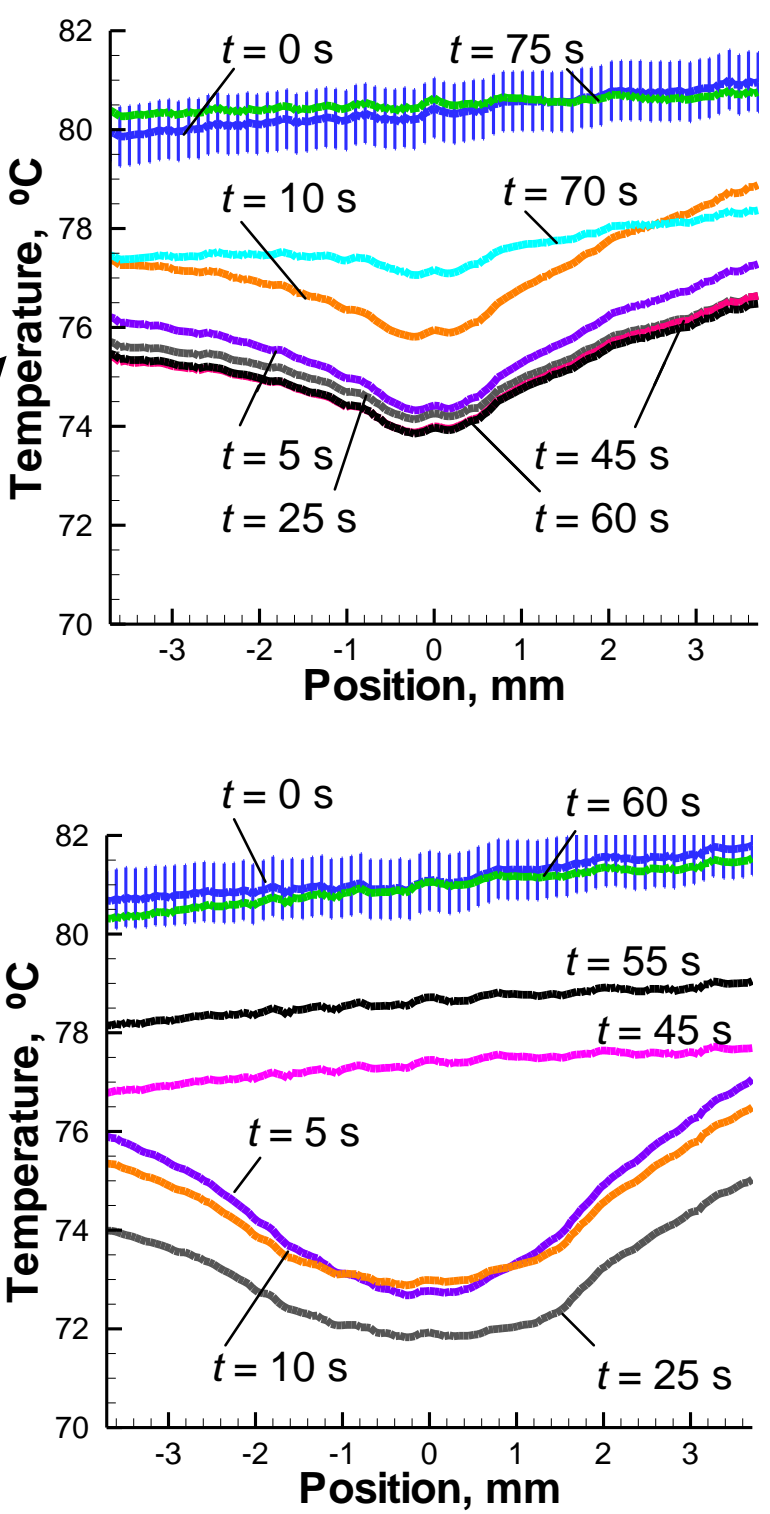

Fig. 5 (Color online) Liquid ribbon cross-sectional shape and device temperature at three times for Surface 3: (a) run without EW actuation $\left(t_{\text {evap }}=69.1 \mathrm{~s}\right)$, and (b) run with EW actuation $\left(t_{\text {evap }}=\right.$ $43.9 \mathrm{~s}$ ). Ribbon volume is $5 \mu \mathrm{L}$. The stripe outline is shown in the first pane of (a). The temperature profiles on the right are taken at the axial midpoint of the stripe. Error bars on the $t=$ $0 \mathrm{~s}$ profile are representative of the confidence in all temperature measurements. 


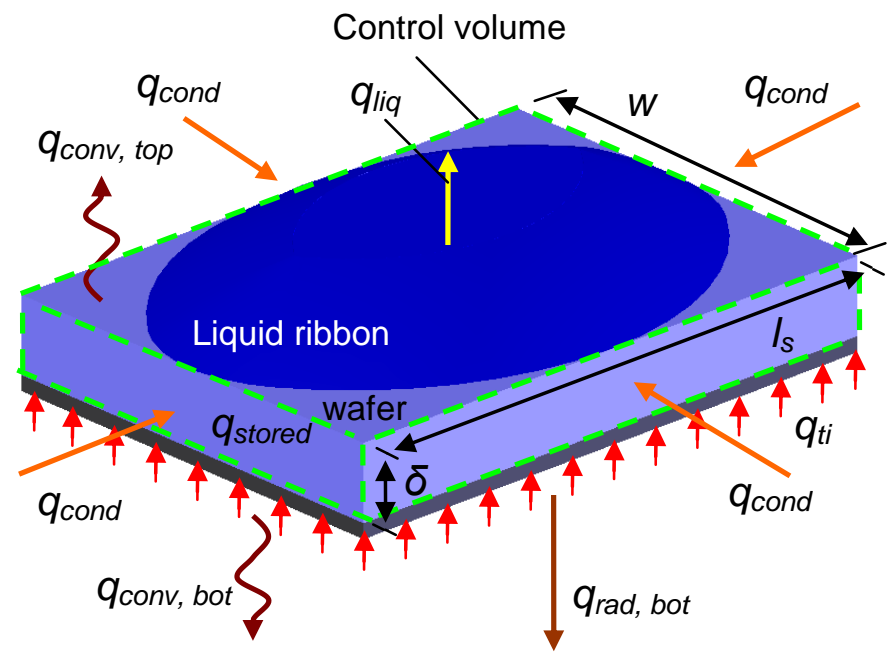

Fig. 6 (Color online) Control volume of device with relevant dimensions and heat fluxes identified. 

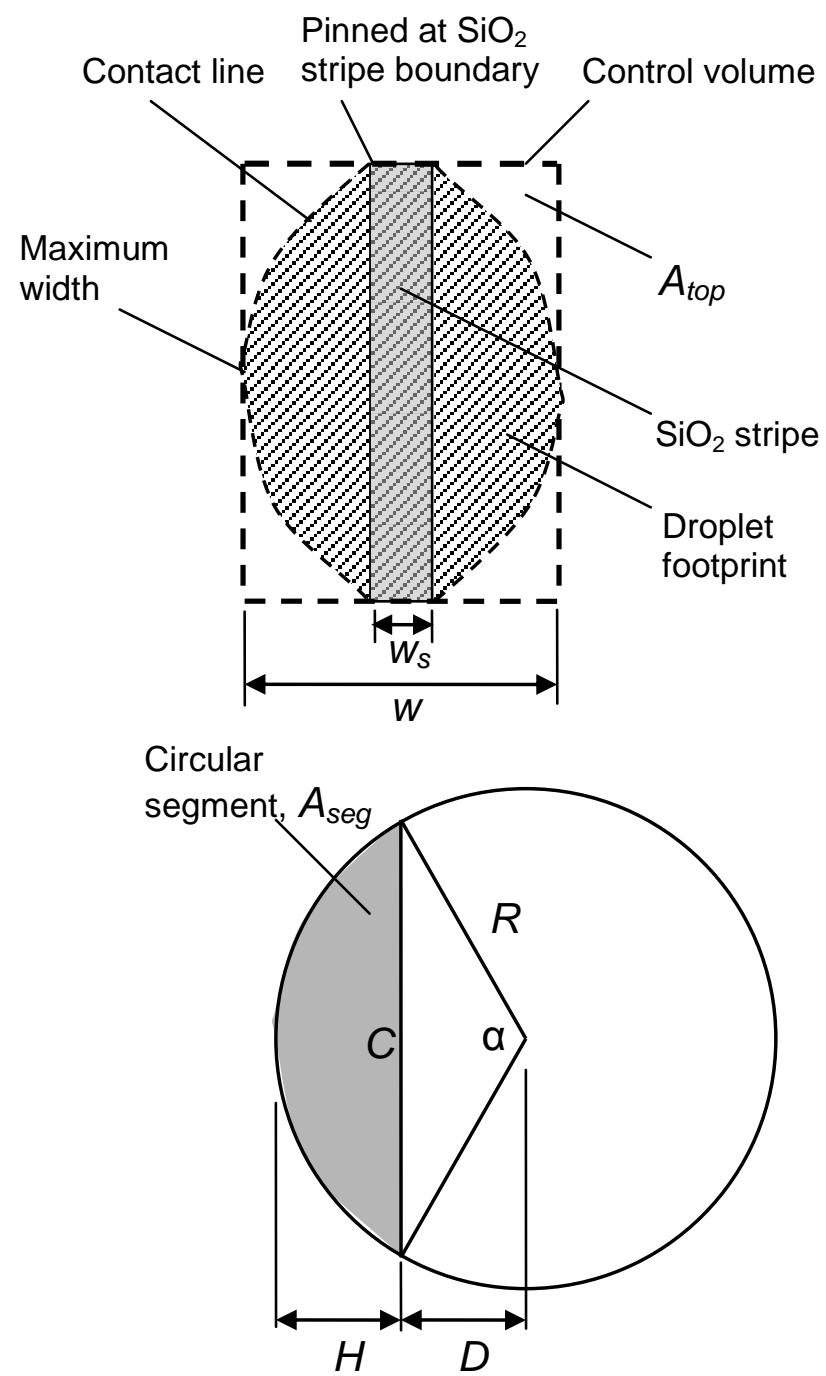

Fig. 7 (Color online) Plan view of control volume illustrating the method used to estimate the exposed top surface area $A_{\text {top }}$. At any given time, the shape of the contact line is assumed to be that of a circular arc between the axial boundaries of the $\mathrm{SiO}_{2}$ stripe and the location of maximum ribbon width. With the area of the resulting circular segment known, $A_{\text {top }}$ can be estimated. 


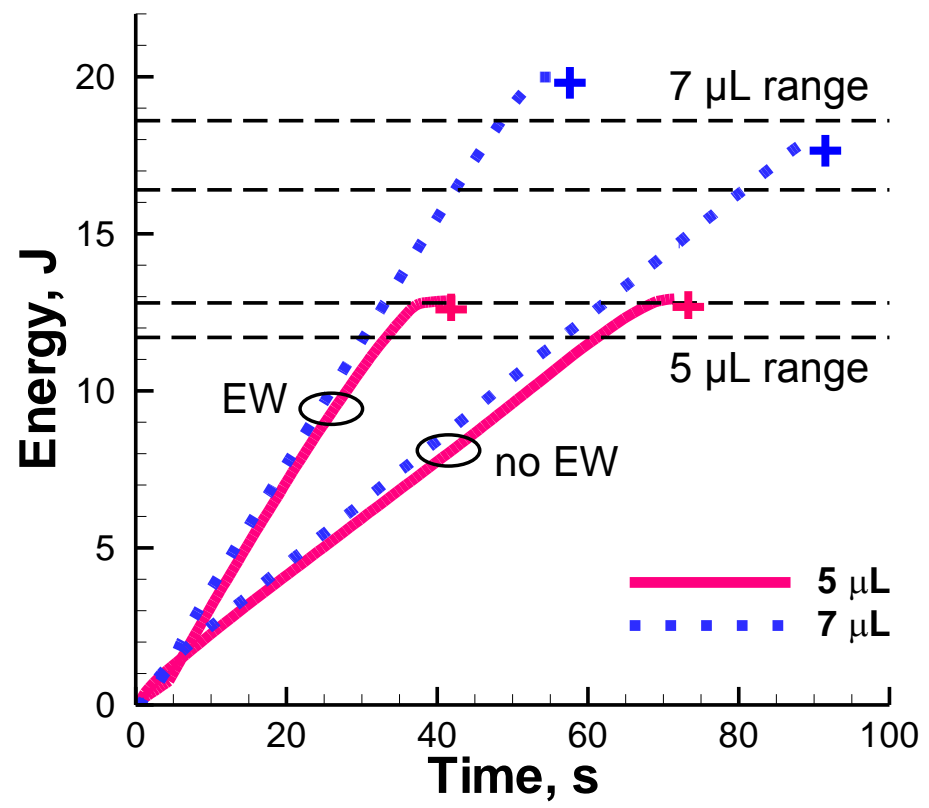

Fig. 8 (Color online) Cumulative energy dissipation $E_{\text {liq }}$ due to 5 and $7 \mu \mathrm{L}$ liquid ribbons as a function of time on Surface 3. + symbols denote time of dry-out. Dashed horizontal lines indicate predicted energy dissipation range. 


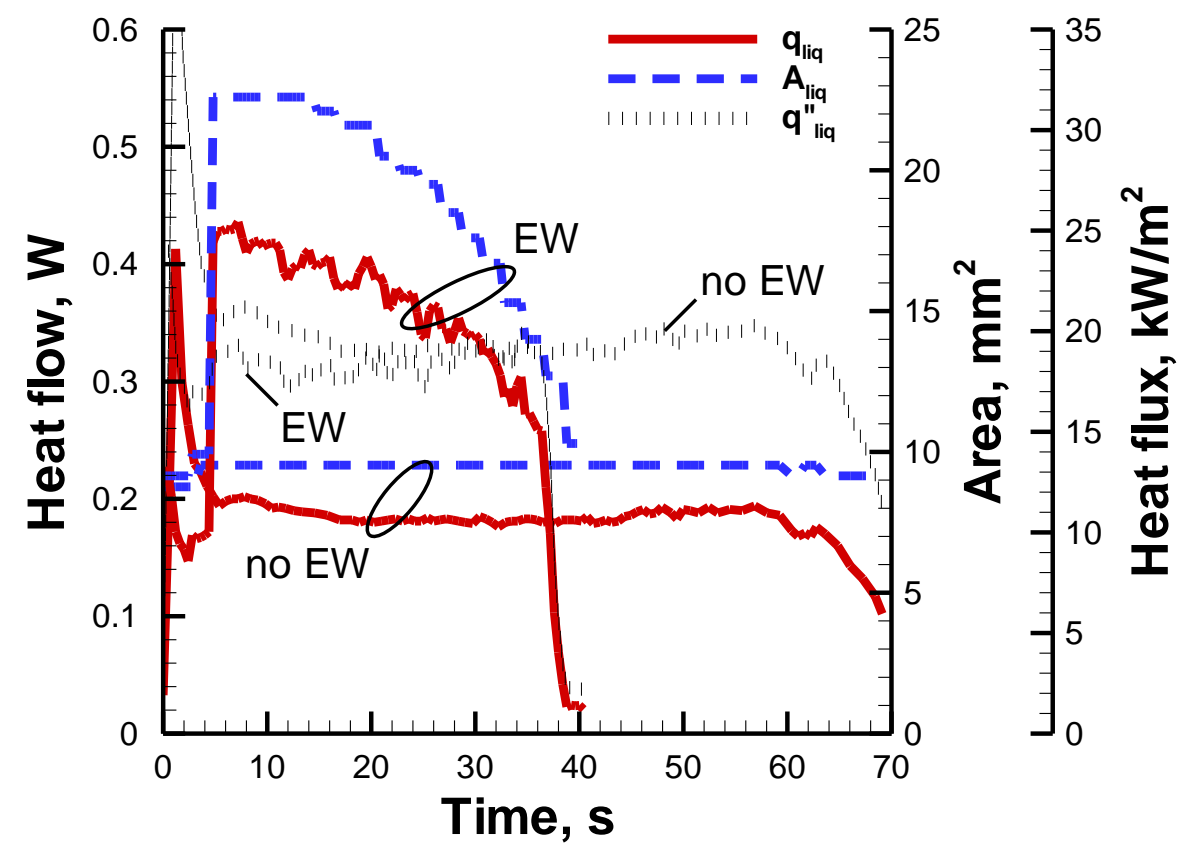

Fig. 9 (Color online) Heat transfer and wetted area of a $5 \mu \mathrm{L}$ liquid ribbon for cases with and without EW on Surface 3. 


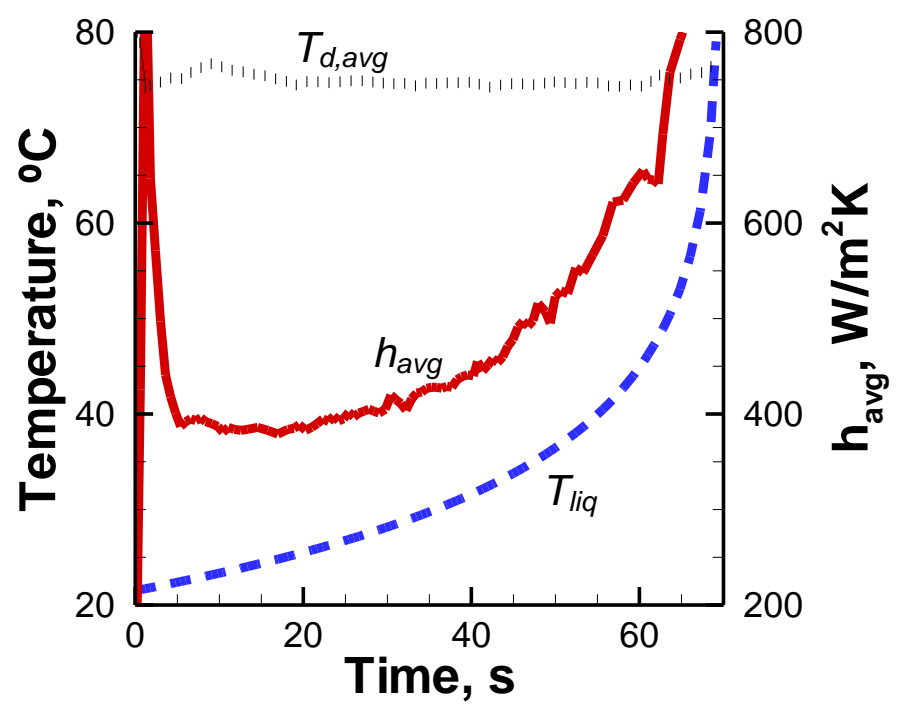

Fig. 10 (Color online) Typical relationship between $T_{\text {liq }}$ and $h_{\text {avg. }} T_{d, a v g}$ is measured while $T_{\text {liq }}$ is estimated based on the assumptions underlying Eqs. (14 - 17). The particular case shown here corresponds to $5 \mu \mathrm{L}$ liquid ribbons on Surface 3 without EW. 


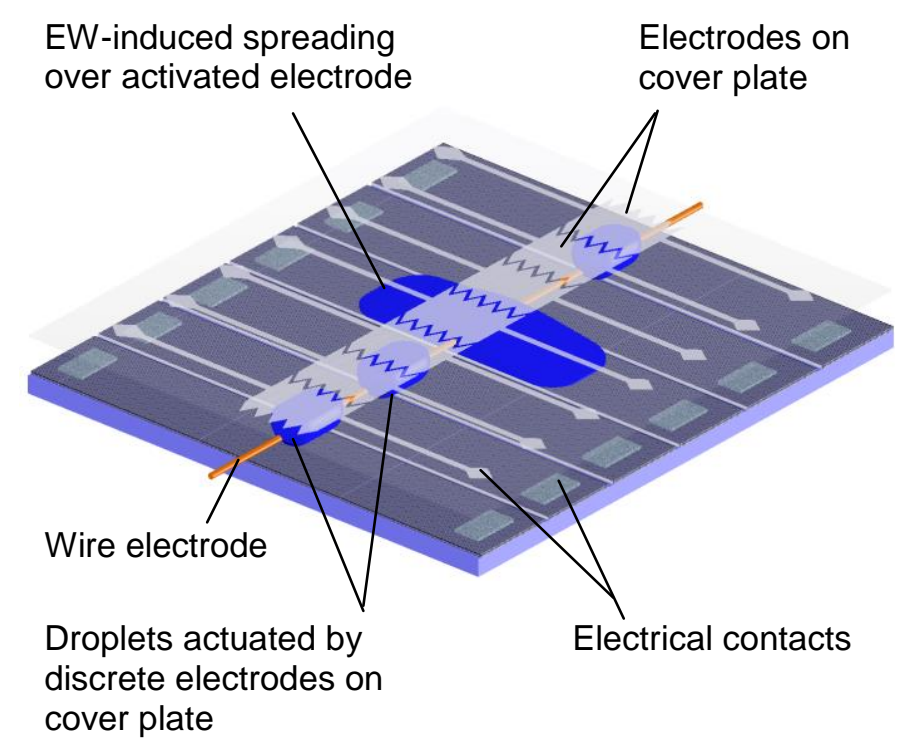

Fig. 11 (Color online) Conceptual illustration of an on-demand device capable of electrowetting a liquid ribbon over a particular surface area via discrete electrodes. The ribbon could be fed by a conventional pump or by electrowetting of another set of electrodes embedded in a cover plate. 